\title{
Combined modulation to incident laser by subsurface crack and contaminant on fused silica
}

Hairong Wang, Zhi Chen, Huapan Xiao, Rongguang Liang, $\mathrm{Na} \mathrm{Yu}$, et al.

Hairong Wang, Zhi Chen, Huapan Xiao, Rongguang Liang, $\mathrm{Na}$ Yu, Jiuhong Wang, "Combined modulation to incident laser by subsurface crack and contaminant on fused silica," Proc. SPIE 10713, Pacific-Rim Laser Damage 2018: Optical Materials for High-Power Lasers, 1071312 (16 May 2018); doi: $10.1117 / 12.2316783$

SPIE Event: Pacific Rim Laser Damage 2018: Optical Materials for High Power Lasers, 2018, Yokohama, Japan 


\title{
Combined modulation to incident laser by subsurface crack and contaminant on fused silica
}

\author{
Hairong Wang ${ }^{* a, b}$, Zhi Chen ${ }^{\mathrm{a}, \mathrm{b}}$, Huapan Xiao ${ }^{\mathrm{a}, \mathrm{b}}$, Rongguang Liang ${ }^{\mathrm{c}}, \mathrm{Na} \mathrm{Ya}^{\mathrm{a}, \mathrm{b}}$, Jiuhong Wang ${ }^{\mathrm{a}, \mathrm{b}}$ \\ ${ }^{a}$ State Key Laboratory for Manufacturing Systems Engineering, Xi'an Jiaotong University, Xi'an, \\ Shaanxi 710049, China; 'School of Mechanical Engineering, Xi'an Jiaotong University, Xi'an, \\ Shaanxi 710049, China; ${ }^{\mathrm{c} C o l l e g e}$ of Optical Sciences, University of Arizona, Tucson, Arizona \\ 85721, USA \\ *whairong@mail.xjtu.edu.cn
}

\begin{abstract}
Subsurface defects and contaminations will be generated during the grinding and polishing processes of optical components. Combined modulation is one of the important factors for the laser-induced damages of fused silica. In this paper, by using 2D finite-difference time-domain method, the light intensity distribution modulated by both radial crack and contaminant is studied on front/rear surface, respectively. The results show that the light intensity distribution is significantly affected by the aspect ratio of radial crack and the relative position between radial crack and contaminant. The simulations of the combined modulation on rear surface show that larger LIEFs are generated at certain relative positions compared with those in the single modulation of radial crack or contaminant. Meanwhile, with the increase of distance, the LIEFs are wave-like up and down fluctuations, and gradually tend to stable values. When there is no total internal reflection, the LIEF in contaminant on the crack wall rises significantly with increase of distance, the maximum LIEF occurs when the contaminant is near the intersecting line between radial crack and rear surface. The simulation of the combined modulation on front surface show that the variation of LIEFs in global domain are not very prominent.
\end{abstract}

Keywords: fused silica, crack, contaminant, light intensity enhancement

\section{INTRODUCTION}

In the high peak power laser system, thousands of optical components like fused silica are required ${ }^{1}$. However, during various optical fabrication processes of cutting, grinding, and polishing, a number of different kinds of defects, (such as contaminants, scratches and cracks etc.) may be introduced into the subsurface layer of optical components ${ }^{2}$. On the one hand, the contaminants with strong sticking are difficult to be completely removed ${ }^{3}$. Due to joint actions of the absorption, scattering and modulation of contaminants, the laser-induced damage has been proved to be an important factor to reduce the reliability and life of lasers ${ }^{4}$. On the other hand, it has already been demonstrated that surface cracks could significantly affect the local fields. Light intensification can result from either discontinuities at crack walls ${ }^{5}$ or constructive interference between the incident beam and the reflected beam ${ }^{6}$. In general, the laser damage of optical component would be caused by contaminants and cracks simultaneously, which hinders its application in the peak power lase system. The optical modulation effects of crack and contaminant on laser have been studied respectively. More specifically, the modulation effect of crack type, size, aspect ratio and position (front or rear surface) on laser has been investigated. Also, the modulation effect of contaminant shape, size, number, dielectric constant and embedded depth on laser has been analyzed. However, the combined modulation effect of crack and contaminant has not been reported.

In this work, the combined model of radial crack and contaminant is established to numerically simulate the light intensity distribution, which is modulated by the radial crack and contaminant on front/rear surface, by using twodimensional finite difference time domain (2D-FDTD) method. The interaction modulation effect between radial crack and contaminant on the laser is analyzed comprehensively. Further, we study the modulation influence of the relative position of crack and contaminant on the laser.

Pacific-Rim Laser Damage 2018: Optical Materials for High-Power Lasers, edited by

Takahisa Jitsuno, Jianda Shao, Wolfgang Rudolph, Proc. of SPIE Vol. 10713, 1071312

(C) 2018 SPIE · CCC code: $0277-786 X / 18 / \$ 18 \cdot$ doi: $10.1117 / 12.2316783$ 


\section{MODEL AND METHOD}

According to Fig. 1, it is found that contaminant often occurs near the crack in fused silica after chemical etching through scanning electron microscopy (SEM) image. The modulation effect of the crack or the contaminant is not enough to explain the modulation effect of subsurface defects on laser ${ }^{2}$. In this case, the combined modulation of crack and contaminant on laser should be considered. For this purpose, the interaction modulation mechanism of crack and contaminant is studied by using 2D-FDTD method. According to simulated results, one can get the light intensity distribution in the vicinity of the crack and contaminant.

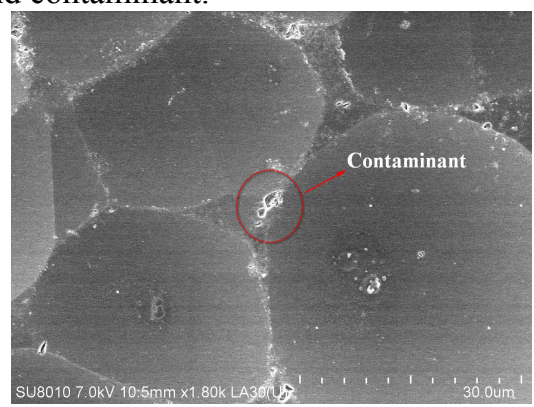

Figure 1. Morphology of cracks and contaminants in fused silica after chemical etching by SEM.

The simulation model for radial crack and contaminant is shown in Fig. 2. The contaminant can be modeled as a sphere of submicron to micron diameter range ${ }^{2}$. Here, a 2D model of crack and contaminant is built under the irradiation of front-surface and rear-surface laser. To simplify the simulation, one radial crack and one circle are selected to represent the crack and contaminant respectively for investigating the combined influence on the laser-induced damage (Fig. 2). The contaminant adheres to the surface or crack wall.

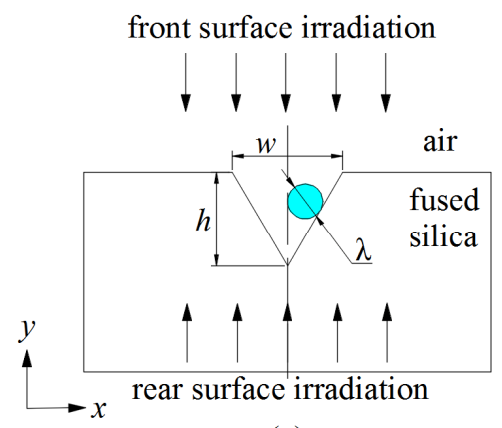

(a)

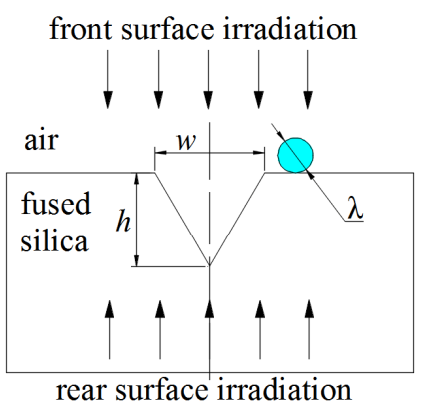

(b)

Figure 2. Schematic diagram of a simulation model: (a) contaminant on the crack wall, (b) contaminant on the surface.

In the simulation, the wavelength of incidence laser is $\lambda=1064 \mathrm{~nm}$. For ensuring the calculation accuracy, the simulation region is meshed by the uniform rectangle with side length $\delta=\lambda / 32=33.25 \mathrm{~nm}$, and the domain size is $640 \delta \times 480 \delta$. The perfectly matched layer (PML) absorbing-boundary condition ${ }^{7-8}$ is utilized in this work. In addition, the laser beam is set with an amplitude of $1.0 \mathrm{~V} / \mathrm{m}$ and its incident direction is along the $y$ axis. Both the transverse electric (TE) wave and the transverse magnetic (TM) wave are considered. The relative dielectric constants of fused silica, air and contaminant are 2.25, 1.0 and 3, respectively. The depth of crack is set as $h=4 \lambda=4.256 \mu \mathrm{m}$, and the radius of contaminant is $0.5 \lambda=0.532 \mu \mathrm{m}$. The time step is determined by adjusting and observing the accurate light intensity distribution within the domain.

The refractive index of fused silica is $n=1.5$. The critical angle of total internal reflection is $41.8^{\circ}$. For the radial crack on rear surface, the incident angle, defined as the angle between the incident wave and the normal of crack wall, is related to the aspect ratio $\xi=h / w$. The incident angles are $75.96^{\circ}, 63.43^{\circ}, 53.13^{\circ}, 45^{\circ}, 38.66^{\circ}, 33.69^{\circ}$, for the cracks with $\xi=4: 2, \xi=4: 4, \xi=4: 6, \xi=4: 8, \xi=4: 10, \xi=4: 12$, respectively. Table 1 shows the relationship between the number of total internal reflection and incident angle 9 .

In this section, the relative position between radial crack and contaminant is changed, and its modulation influence on the laser is investigated. Light intensity enhancement factor (LIEF) is introduced to quantitatively characterize the localized 
light intensity distribution caused by surface crack and contaminant ${ }^{10-12}$. The LIEF is defined as maximal light intensity enhancement caused by surface cracks and contaminants in the certain domain.

Table 1. The relationship between the number of total internal reflection and incident angle ${ }^{9}$.

\begin{tabular}{ccc}
\hline Incident angle $\theta$ & Aspect ratio $\xi$ & $\begin{array}{c}\text { Number of total internal } \\
\text { reflections }\end{array}$ \\
\hline $0<\theta<41.8^{\circ}$ & $4: 10 ; 4: 12$ & 0 \\
$41.8^{\circ} \leq \theta \leq 45^{\circ}$ & $4: 8$ & 1 \\
$45^{\circ}<\theta \leq 69.1^{\circ}$ & $4: 4 ; 4: 6$ & 2 \\
$\theta>69.1^{\circ}$ & $4: 2$ & 1 \\
\hline
\end{tabular}

\section{RESULTS AND DISCUSSION}

\subsection{Combined modulation of radial crack and contaminant on rear surface}

Figures 3(a) and 3(b) show the light intensity distribution modulated by both one crack and one contaminant on rear surface under TE mode illumination. The aspect ratio of crack is $\xi=4: 4$, and the angle of incidence is $63.43^{\circ}$. The incident beam can be totally internally reflected by the crack and then by rear surface. In Figs. 3(a) and 3(b), the contaminants are on the crack wall and on rear surface, respectively. Figures 3(c) and 3(d) show the light intensity distribution modulated by only one radial crack and one contaminant, respectively. All geometric parameters of crack and contaminant are the same in Fig. 3.

According to results, when the contaminant is on the crack wall (Fig. 3(a)), the LIEF in global domain is 8.63, locating in the fused silica. It approximately equals to the LIEF in the global domain with only one radial crack (Fig. 3(c)), which is 8.36. The light intensity distributions in fused silica seem similar in these two cases. It means that the contaminant may be negligible for the modulation on laser in fused silica. The light intensity distribution in fused silica is the result of the interference between the incident beam and total internal reflected beam at the crack wall and rear surface ${ }^{13}$. The LIEF in contaminant is only 1.51 . It can be primarily attributed to the totally internally reflection at the crack wall, and in such a case the incident beam barely passes through the crack wall to get into the contaminant. When the contaminant is on rear surface (Fig. 3(b)), the LIEF in global domain is 13.62, locating in the contaminant. The LIEF value is larger than that with only one contaminant, which is 8.53 (Fig. 3(d)). There is a strong beam going through the location of contaminant (see Fig. 3(c)), which focuses within the contaminant to generate an enhanced light intensity. The LIEF in fused silica is 10.34, which is larger than that with only one radial crack. And the position of the LIEF in fused silica is near the contaminant, which is caused by the interference between the light reflected from contaminant and light in fused silica. In general, the combined modulation between radial crack and contaminant can play a co-effect on the light intensity distribution around radial crack and contaminant, resulting in an enhanced LIEF eventually.

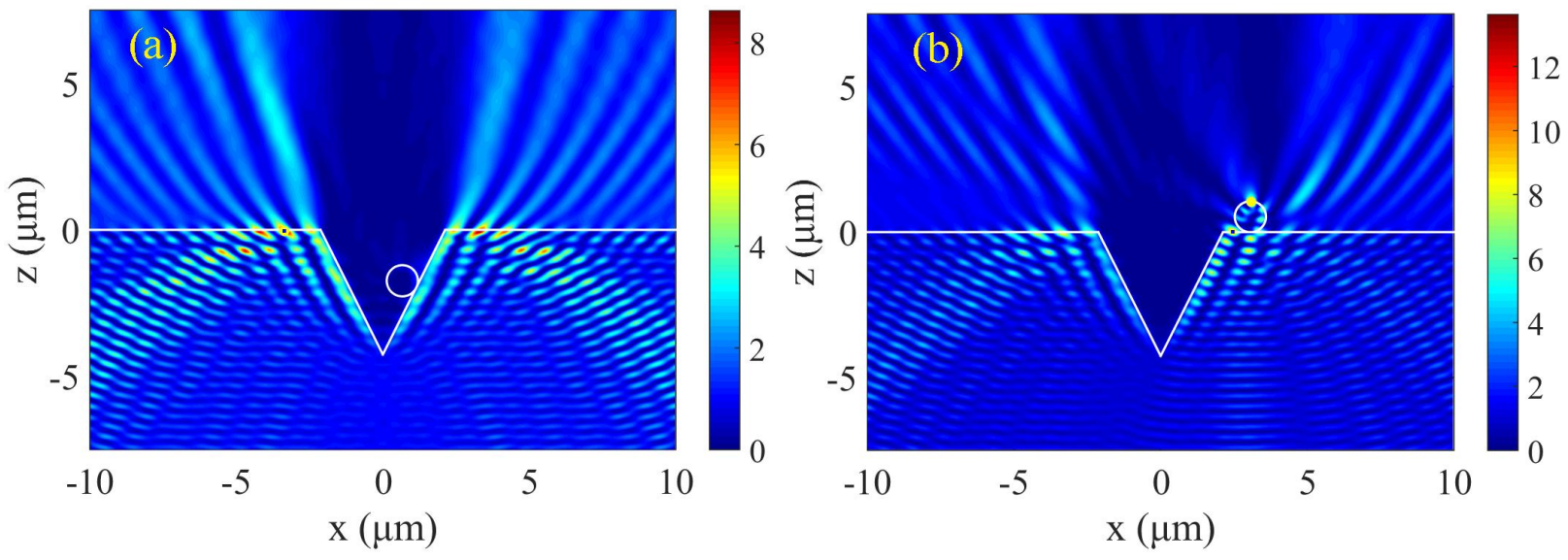



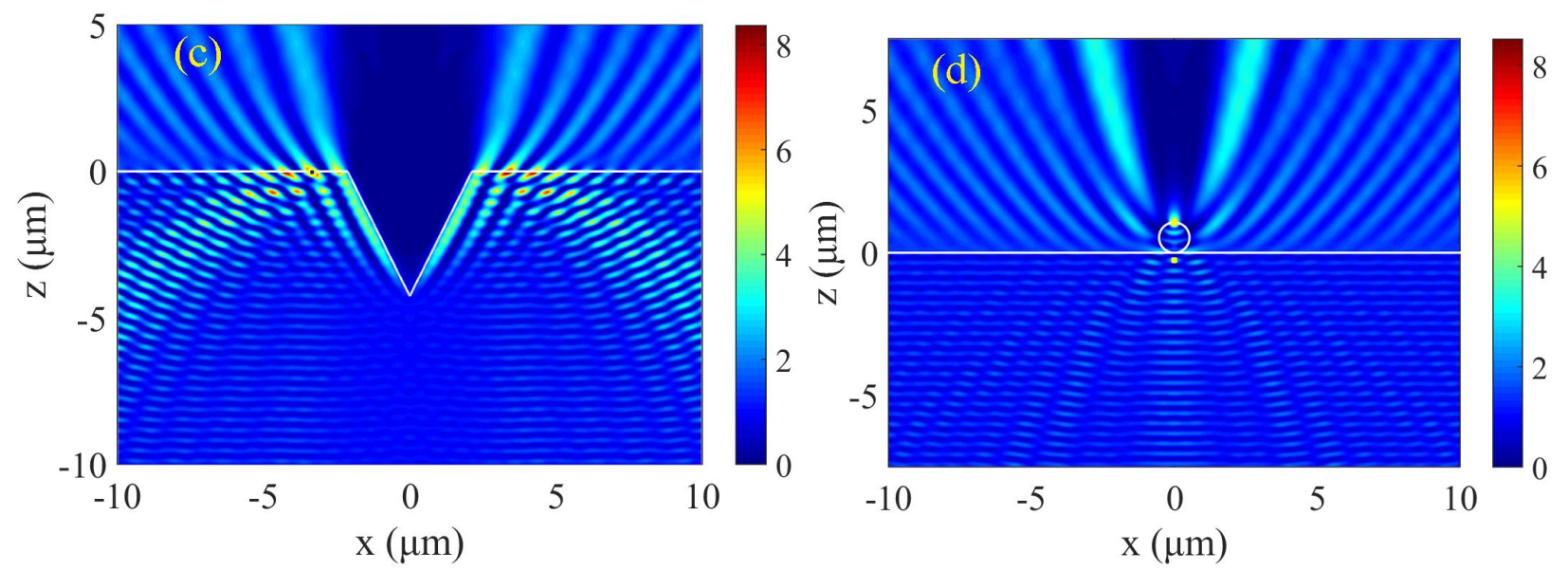

Figure 3. Light intensity distribution modulated by the radial crack and contaminant on rear surface under the TE mode illumination. (a) contaminant on the crack wall, (b) contaminant on rear surface, (c) only the radial crack, (d) only the contaminant.

Figure 4 shows the variation of LIEFs in certain domains (global domain, fused silica domain and contaminant domain) with respect of the related positions between radial crack and the contaminant on rear surface under TE mode and TM mode illumination. The $x$ axis is the distance from the contaminant to the center axis of radial crack. From the results with different aspect ratios of radial cracks, as shown in Figs. 4(a)-4(f), three disciplines can be summed. First, the LIEFs in global domain under TE mode illumination are generally in fused silica or contaminant, while they are almost in the air under TM mode illumination. Second, when the contaminant is on rear surface, as the contaminant moves away from the crack, the LIEFs in each domain under TE mode or TM mode illumination are wave-like up and down fluctuations, and gradually tend to stable values. Figure 3(c) shows that the light wave around the crack is modulated into the approximately parallel stripes by radial crack, and these stripes alternate with strong and weak light intensity. When the contaminant is in a strong light intensity band, most of light enter the contaminant and are focused into a stronger light. After passing through the contaminant, the light interferes with the light that exists in the air. The light reflected by contaminant enters into the fused silica, and it interferes with the light that exists in fused silica. And the LIEF in fused silica is near the contaminant. So the larger LIEF may appear in each domain. Conversely, in a weak light intensity band, less LIEF appears in each domain. As the contaminant moves away from the crack, the coupling effect becomes weaker and weaker. Namely, the crack and contaminant modulate the laser respectively, finally the LIEFs in each domain tend to stable values. Third, as for the contaminant on the crack wall, with the increase of $x$ value, the LIEF in contaminant decreases a little, and then increases gradually. Figure 4(e) shows this phenomenon. When the contaminant is at the position $x=0$, the light passes through the two crack walls of radial crack, enters into the contaminant, and is focused. As the contaminant moves along the crack wall, less light that transmitted from the other crack wall enters into the contaminant. So the LIEF decreases a little first. Figure 5 shows the light intensity distribution around the slope at the same incident angle with radial crack in Fig. 4(e). The light beam passing through the slope inclines toward the slope, which is consistent with the Snell's law. The light intensity increases along the slope. So the stronger light enters into contaminant on the crack wall, and the LIEF increases gradually along the crack wall. By the way, because there is little light through the totally internally reflected crack wall, the LIEF in contaminant is very small. But it still meets this discipline (Figs. 4(a)-4(d)).

The red horizontal dashed line indicates the LIEF in fused silica with only one radial crack, and the blue horizontal dashed line indicates the LIEF in contaminant with only one contaminant. When the double total internal reflections occur, the LIEFs in fused silica are relatively large, as shown in Figs. 4(b) and 4(c). However, as Figs. 4(e) and 4(f) shown, when no total internal reflection occurs, the LIEFs in fused silica are relatively small. This is consistent with the reported light intensity distribution caused by rear-surface features ${ }^{13}$. In Figs. 4, most of the corresponding curves are above the two horizontal dashed lines. It means that compared with the single modulation of radial crack or contaminant, much larger LIEFs are generated due to the combined modulation at certain relative positions. The black vertical dashed line represents the intersecting line between the crack surface and rear surface of fused silica. From Figs. 4(e) and 4(f), when the contaminant is near the intersecting line between rear surface and the crack with a relatively small aspect ratio, the LIEF appears to have a maximum value. This result can explain the experimental phenomenon that crack boundary is 
more susceptible to suffer from laser-induced damage ${ }^{14}$. In a word, the relative position between radial the crack and the contaminant on rear surface is a significant influence factor for the combined modulation on laser.
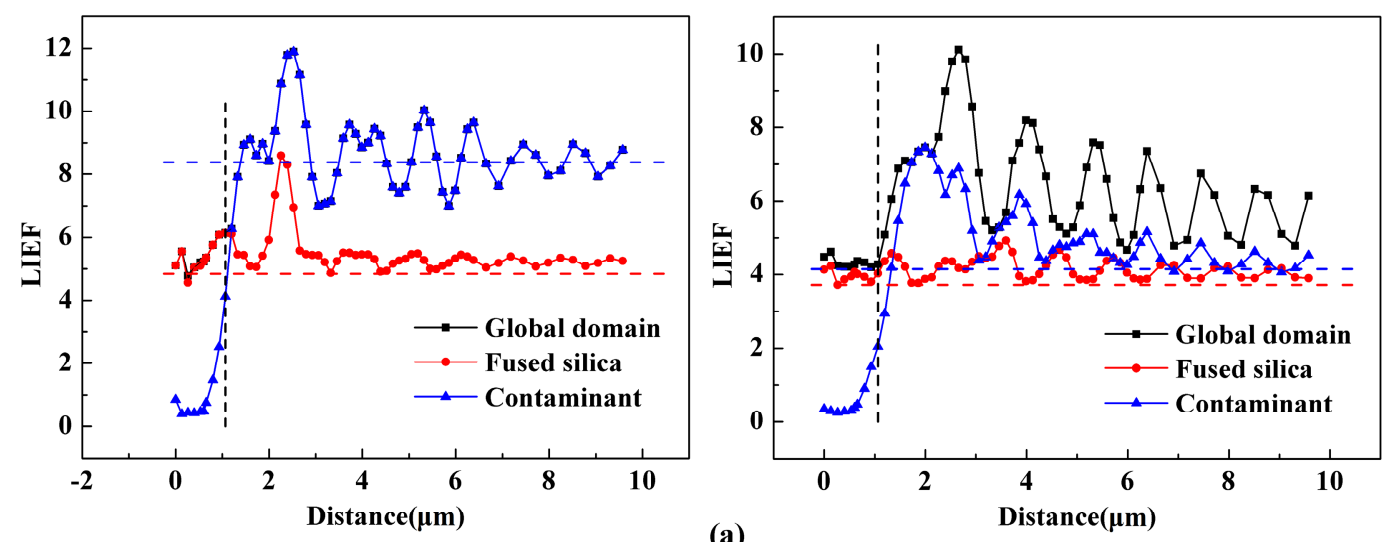

(a)
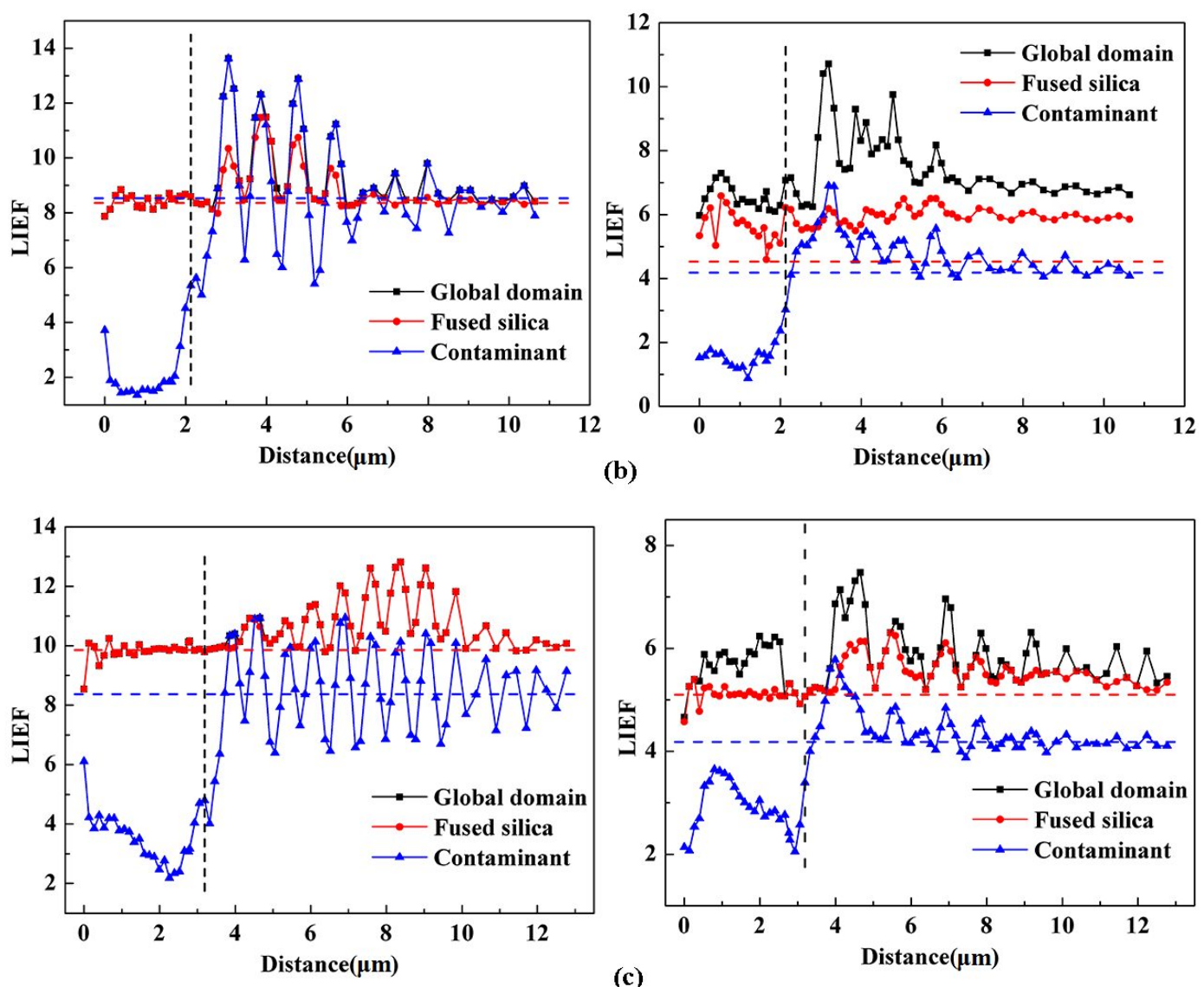

(c) 

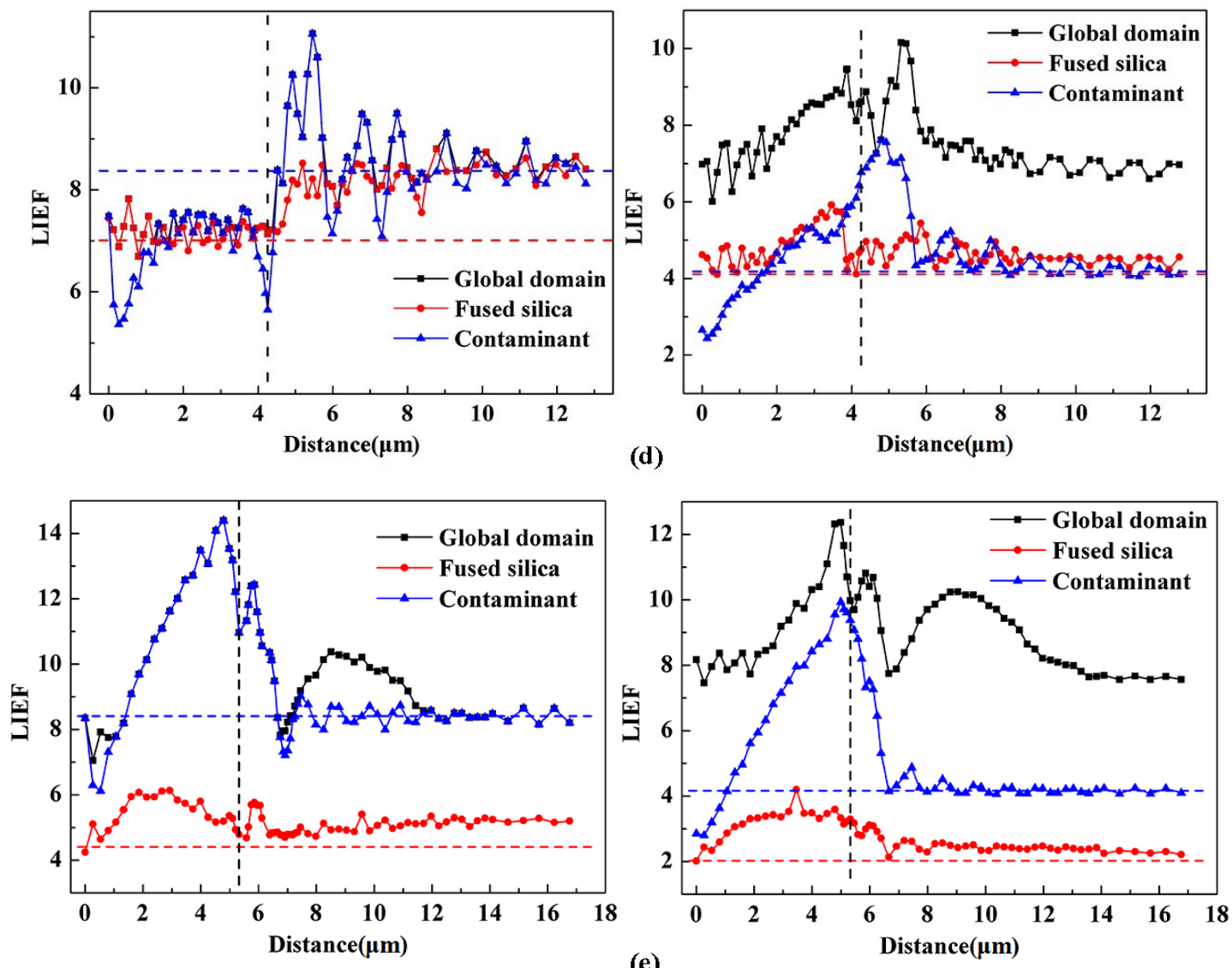

(e)
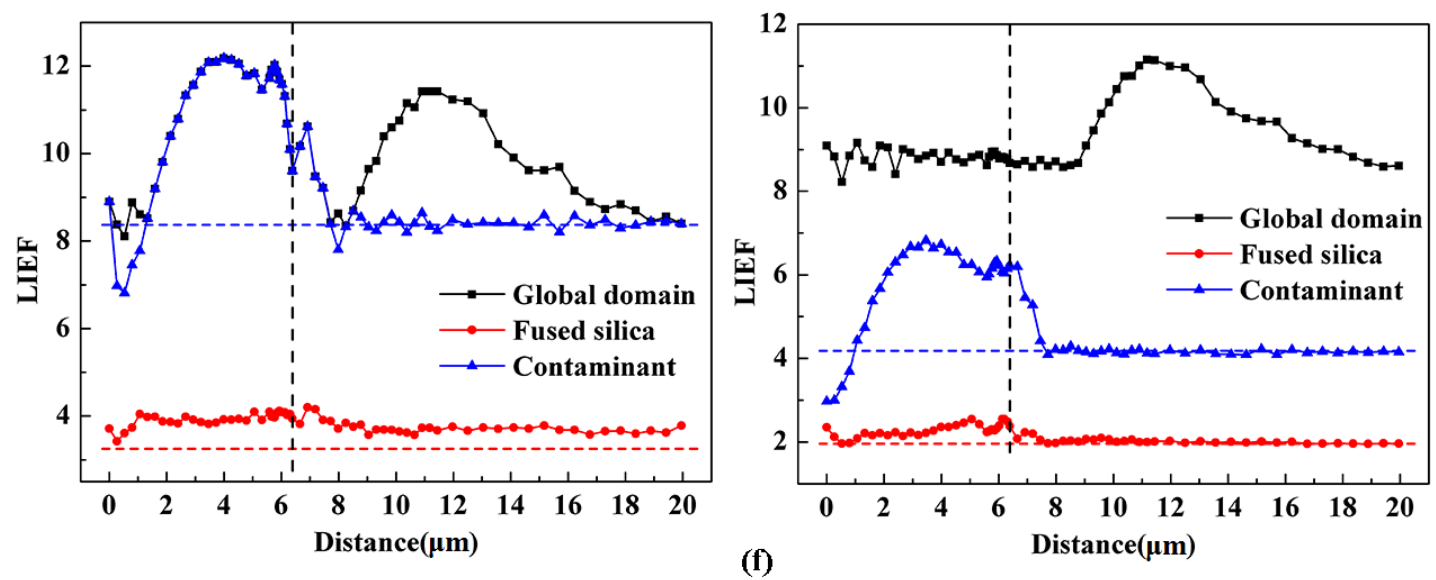

Figure 4. Variation of LIEFs in global domain, fused silica and contaminant with respect of the related positions between the radial crack and the contaminant on rear surface. Aspect ratio of radial crack is (a) $\xi=4: 2$, (b) $\xi=4: 4$, (c) $\xi=4: 6$, (d) $\xi=4: 8$, (e) $\xi=4: 10$, (f) $\xi=4: 12$. Left: under TE mode illumination; right: under TM mode illumination. 


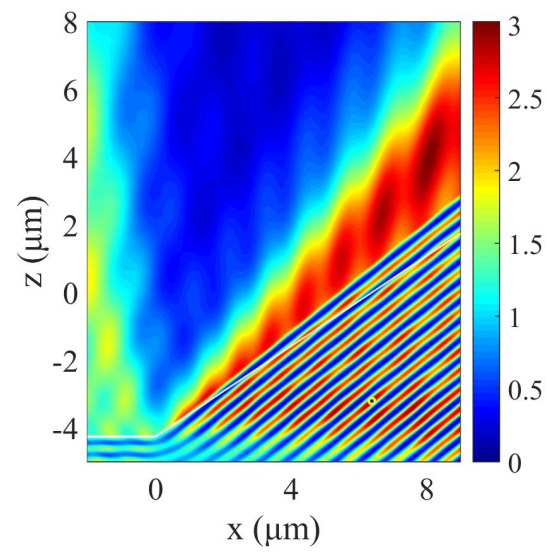

Figure 5. Light intensity distribution around the slope on rear surface under TE mode illumination (Incident angle is $38.66^{\circ}$ ).

\subsection{Combined modulation of radial crack and contaminant on front surface}

Figures 6(a) and 6(b) show the light intensity distribution modulated by both one crack and one contaminant on front surface under TE mode illumination. Figures 6(e) and 6(f) show the light intensity distribution modulated by both one crack and one contaminant on front surface under TM mode illumination. The crack width is $w=5 \lambda=5.32 \mu \mathrm{m}$. In Figs. 6(a) and 6(e), the contaminant is on the crack wall, while it is on front surface in Figs. 6(b) and 6(f). Figs. 6(c) and 6(d), $6(\mathrm{~g})$ and $6(\mathrm{~h})$ show the light intensity distribution modulated by only one radial crack and one contaminant, respectively. All geometric parameters of crack and contaminant are the same in Fig. 6.

For the radial crack on front surface under TE mode illumination, the light intensification is generated through three physical mechanisms: (1) diffraction originated from the intersection point of crack wall and front surface, (2) interference between reflected light inside the crack and incident light, and (3) interference ripples between transmitted light from both the crack walls and front surface (Fig. 6(c) $)^{13}$. In crack gap, some discrete hot spots are generated due to the interference. The LIEF in global domain is 3.49. In Fig. 6(a), the incident light and reflected light on the crack wall enter into the contaminant and are focused. The LIEF in contaminant is 6.31 , which is almost twice as the LIEF with only one radial crack. For the contaminant on front surface, the light intensity distribution around the contaminant is similar to that without the crack (Figs. 6(b) and 6(d)). The LIEF is 3.80 in these two cases. This is because the crack has little effect on the light intensity distribution of front surface (see in Fig. 6(c)). For the LIEF in fused silica, it is 3.13 with only one radial crack. When the contaminant is on the crack wall, the LIEF is 3.82 , while when the contaminant is on front surface, the LIEF is 4.26. The reason is that the light passing through the contaminant enters the fused silica, and interferes with the transmitted light from both crack wall and front surface. However, the difference of the relative position of contaminant and crack leads to different results of interference. For the radial crack on front surface under TM mode illumination, the light distribution is similar with that under TE mode illumination. But the LIEF is smaller than that under TE mode illumination. Particularly, due to the small LIEF in crack gap (Fig. 6(g)), when the contaminant is on the crack wall (Fig. 6(e)), the LIEF is still small. In general, the light intensity distribution in fused silica is slightly affected due to the contaminant.
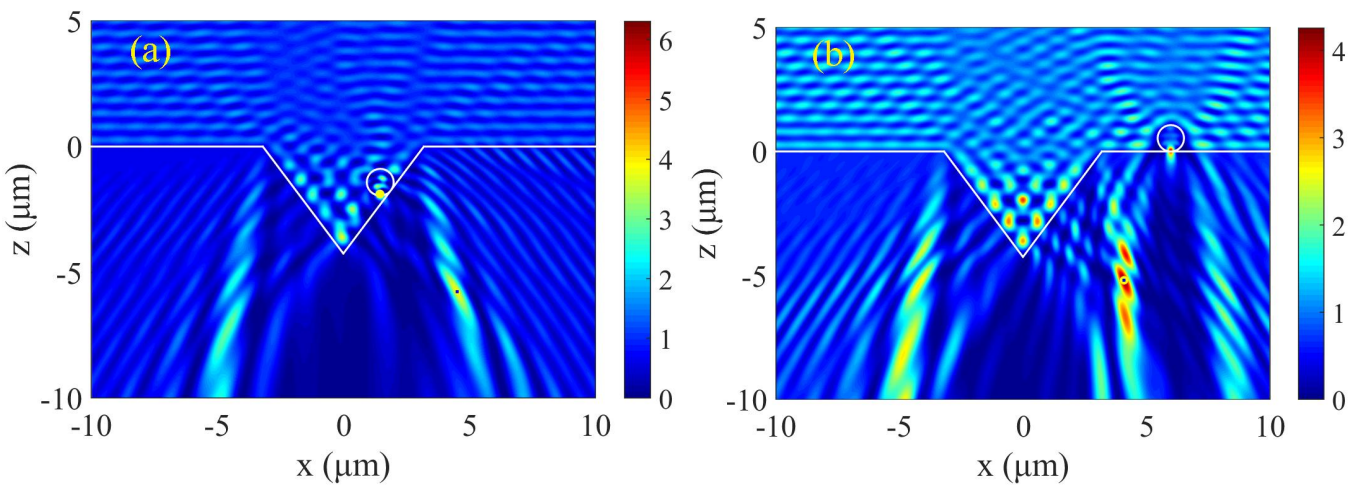

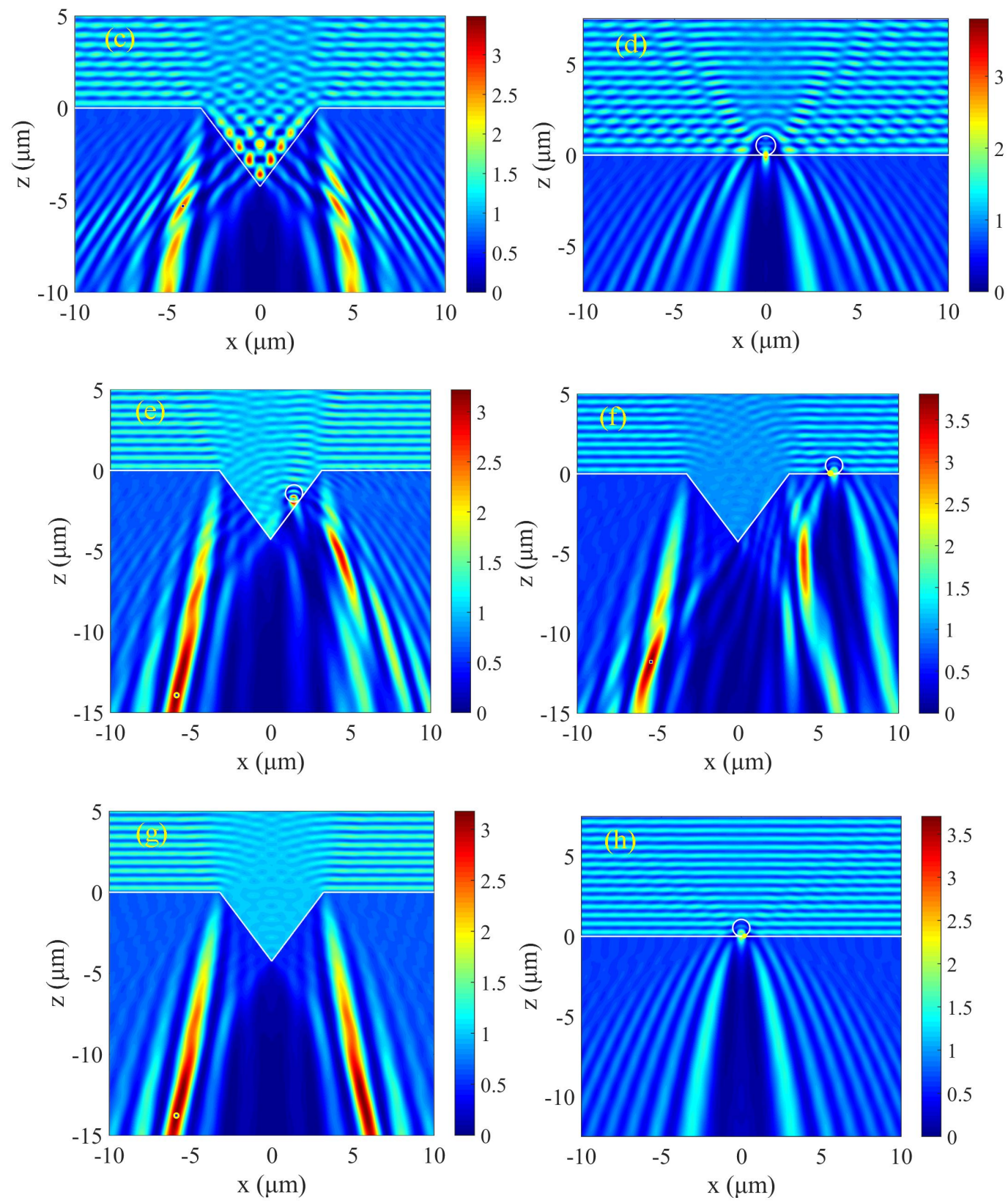

Figure 6. Light intensity distribution modulated by the radial crack and contaminant on front surface. (a) and (e) contaminant on the crack wall, (b) and (f) contaminant on rear surface, (c) and (g) only one radial crack, (d) and (h) only one contaminant, (a-d) under TE mode illumination, (e-h) under TM mode illumination.

Figure 7 shows the variation of LIEFs in certain domains (global domain, fused silica domain and contaminant domain) with respect of the related positions between the radial crack and the contaminant on front surface under both TE mode and TM mode illumination. In Figs. 7(a) and 7(b), the aspect ratio is $\xi=4: 6, \xi=4: 8$, respectively. The curves in Figs. 7 (a) and 7(b) have the same trend. It means that the aspect ratio of radial crack has little effect on the LIEF. When the contaminant is on the crack wall, the variation of the LIEF in the contaminant under TE mode illumination are wave-like up and down fluctuations, and the LIEFs in the contaminant are larger than that without the contaminant. This is because there are some discrete hot spots near the crack wall (Fig. 6(c)). As the contaminant moves along the crack wall, the strong light intermittently enters the contaminant and is focused into stronger light. When the contaminant is near the intersecting line between the crack and front surface, the LIEF drops to about 3.8. As mentioned before, the crack has 
little effect on the light intensity distribution of front surface. The LIEF keeps at 3.8 as the contaminant moves on front surface. The light focused by the contaminant enters the fused silica and interferes with light transmitted from the crack wall and front surface. The LIEF in fused silica fluctuates with the change of the position of contaminant. However, it does not increase with a large amplitude.

The variations of LIEFs under TM mode illumination are similar to those under TE mode illumination. Compared with that under TE mode, the LIEFs under TM mode are smaller. As the light intensity in the crack gap is weak, as shown in Fig. 6(g), when the contaminant is assumedly put on the crack wall, the LIEF in the contaminant is small naturally. When the contaminant is near the intersecting line between the crack surface and front surface, the maximum LIEF in global domain happens in the air, which is marked by dashed line, as shown in right diagram of Fig. 7(a).
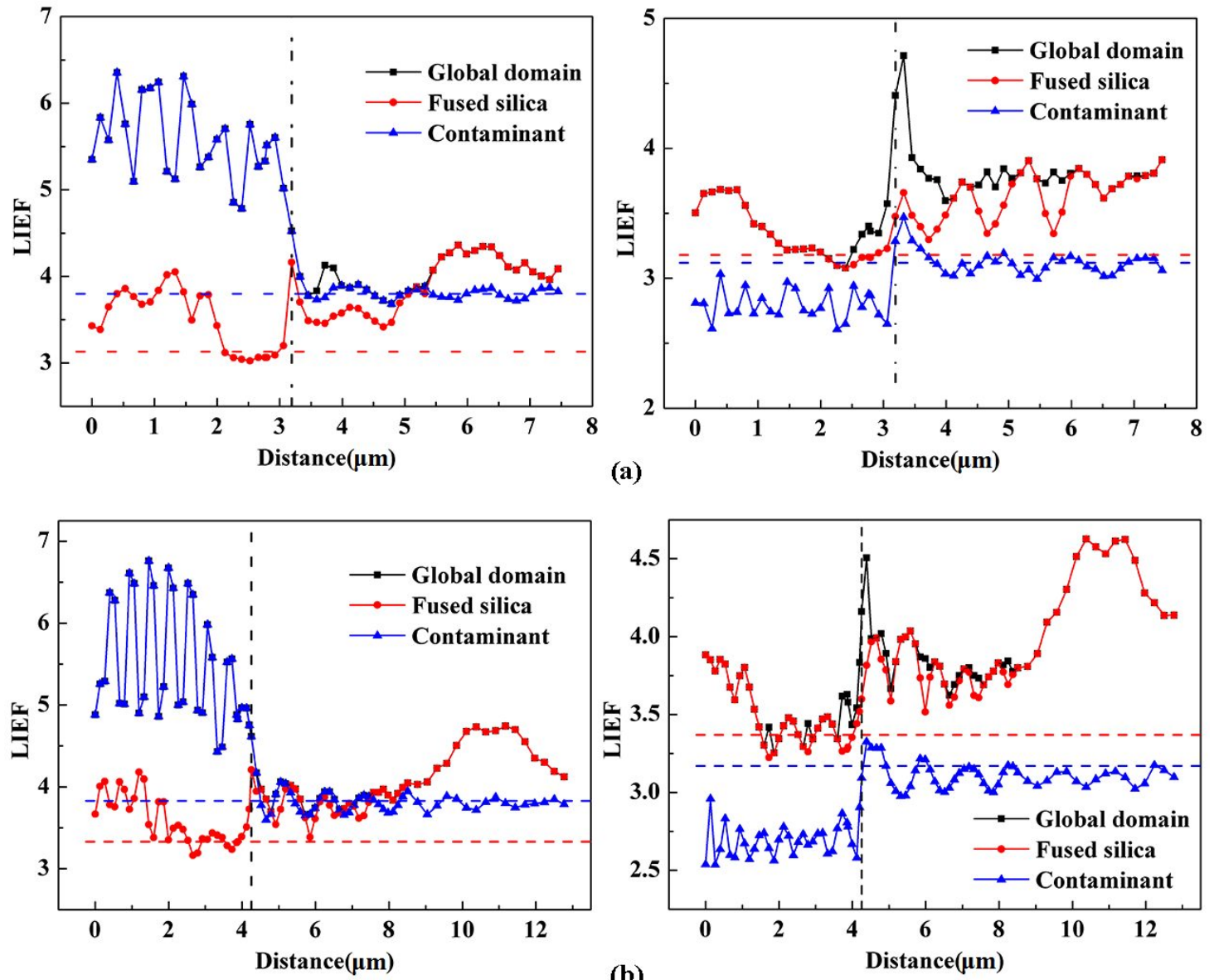

Figure 7. Variations of LIEFs in global domain, fused silica and contaminant with respect of the related positions between the radial crack and the contaminant on front surface. Aspect ratio of radial crack is (a) $\xi=4: 6$ and (b) $\xi=4: 8$. Left: TE mode illumination, right: TM mode illumination.

\section{CONCLUSIONS}

In this paper, the light intensity distribution resulting from the combined modulation between the radial crack and the contaminant has been simulated. The results show that the light intensity distribution is significantly affected by aspect ratio of radial crack and the relative position between radial crack and the contaminant. The simulations of the combined modulation on rear surface show that compared with the single modulation of radial crack or contaminant, much larger LIEFs are generated due to the combined modulation at certain relative positions. Meanwhile, as the contaminant on rear surface moves away from the crack, the LIEFs under TE mode or TM mode illumination are wave-like up and down fluctuations, and gradually tend to stable values. In addition, when there is no total internal reflection, the LIEF in contaminant on the crack wall rises significantly with increase of distance, and the maximum LIEF occurs when the 
contaminant is near the intersecting line between radial crack and rear surface. The simulations of the combined modulation on front surface show that the variation of LIEFs in global domain are not very prominent. This work is to provide a reference for analyzing the effect of subsurface defects on laser-induced damage of optical components.

\section{ACKNOWLEDGMENT}

This work is supported by the National Natural Science Foundation of China (Grants No. 51175416 and No. 51675420), 111 Program (Grant No. B12016) and the National Key Research \& Development (R\&D) Program of China (Grant No. 2016YFB0501604-02). We appreciate the support from the International Joint Laboratory for Micro/Nano Manufacturing and Measurement Technologies.

\section{REFERENCES}

[1] Campbell, J. H., Hawley-Fedder, R. A., Stolz, C. J., Menapace J. A., Borden M. R., Whitman P. K., Yu J., Runkel M. J., Riley M. O., Feit M. D. and Hackel R. P., "NIF optical materials and fabrication technologies: an overview," Proc. SPIE 5341, 84-101 (2004).

[2] Zhang, L., Chen, W., Hu, L., "Systematic investigation on light intensification by typical subsurface cracks on optical glass surfaces," Appl. Opt. 52(5), 980-989 (2013).

[3] Yang, L., Xiang, X., Miao, X. X., Yuan, X. D., Li, L., Yan, Z. H., Zhou, G. R., Lv, H. B., Zheng, W. G., Zu, X. T., "Numerical simulation of modulation to incident laser by submicron to micron surface contaminants on fused silica," Chin. Phys. B 25(1), 014210 (2016).

[4] Feit, M. D., Rubenchik, A. M., Kozlowski, M. R., "Surface-contamination-initiated laser damage," Proc. SPIE 3047, 480-488 (1997).

[5] Bloembergen, N., "Role of cracks, pores, and absorbing inclusions on laser induced damage threshold at surfaces of transparent dielectrics," Appl. Opt. 12, 661-664 (1973).

[6] Génin, F. Y., Salleo, A., Pistor, T. V., and Chase, L. L., "Role of light intensification by cracks in optical breakdown on surfaces," Opt. Soc. Am. A 18, 2607-2616 (2001).

[7] Oubre, C. and Nordlander, P., "Optical properties of metallodielectric nanostructures calculated using the finite difference time domain method," J. Phys. Chem. B 108(46), 17740-17747 (2004).

[8] Li, L., Xiang, X., Zu, X. T., Yuan, X. D., He, S. B., Jiang, X. D., Zheng, W. G., "Incident laser modulation of a repaired damage site with a rim in fused silica rear subsurface," Chin. Phys. B. 21(4), 351-356 (2012).

[9] Xiao, H. P., Wang, H. R., Chen, Z., Fu, G. L., and Wang, J. H., "Effect of brittle scratches on transmission of optical glass and its induced light intensification during the chemical etching," Opt. Eng. 56(10), 105101 (2017).

[10] Génin, F. Y., Salleo, A. T., Pistor, V., and Chase, L. L., "Role of light intensification by cracks in optical breakdown on surfaces," J. Opt. Soc. Am. A 18(10), 2607-2616 (2001).

[11]Zhang, L., Chen, W., and Hu, L., "Systematic investigation on light intensification by typical subsurface cracks on optical glass surfaces," Appl. Opt. 52(5), 980-989 (2013).

[12] Cheng, J., Chen, M., Liao, W., Wang, H., Xiao, Y., and Li, M., "Fabrication of spherical mitigation pit on KH2PO4 crystal by micro-milling and modeling of its induced light intensification," Opt. Express 21(14), 16799-16813 (2013).

[13] Cheng, J., Chen, M. J., Liao, W., Wang, H. J., Wang, J. H., Xiao, Y., and Li, M. Q., "Influence of surface cracks on laser-induced damage resistance of brittle KH2PO4 crystal," Opt. Express 22(23), 28740-28755 (2014).

[14] Suratwala, T. I., Miller, P. E., Bude, J. D., Steele, W. A., Shen, Nan., Monticelli, M. V., Feit, M. D., Laurence, T. A., Norton, M. A., Carr, C. W., and Wong, L. L., "HF Based Etching Processes for Improving Laser Damage Resistance of Fused Silica Optical Surfaces,” J. Am. Ceram. Soc. 94(2), 416-428 (2011). 